\title{
Auditoria em contas médicas no Hospital da Polícia Militar de Pernambuco
}

\author{
Grace Mary Gomes Cintra ${ }^{1}$; Carla Giselly de Souza ${ }^{2}$; Marta Gerusa Silva de Souza ${ }^{3}$
}

\begin{abstract}
Resumo: A auditoria em Serviços de Saúde é um tema dos mais relevantes às Instituições de Saúde, porquanto essencial à manutenção da própria "saúde financeira" de tais organizações. Deste modo, para manterem-se no mercado competitivo, as instituições de saúde têm que aprender a associar baixos custos com excelência de qualidade para os seus pacientes. A auditoria de enfermagem representa a função de controle do processo administrativo, verificando se os resultados da assistência estão de acordo com os objetivos. Deste modo esta pesquisa objetivou avaliar o valor total de contas mensal, das principais clinicas credenciadas ao HPMPE e os principais atendimentos realizados com: atendimento ambulatorial, internamentos e exames. A pesquisa foi realizada no setor de Contas médicas, onde se avaliou as contas recebidas para auditoria no referido hospital no mês de agosto de 2010 nas unidades da capital e do interior. O critério de pesquisa pautou-se pela instituição credenciada, valor cobrado, valor glosado, valor pago, motivo da glosa e o número total de contas auditadas. Durante este período quantificou-se um total geral de contas pagas de R\$ 1.483.474,02 na capital e interior do estado de Pernambuco, deste foi gerada uma glosa de R\$ 63.826,87. Para que possamos diminuir o percentual total de perdas hospitalares precisamos que enfermeiros e instituições avaliem como estão sendo realizados os registros de enfermagem, tendo uma visão crítica e propositora de sua atuação, pois devemos desempenhar as atividades focando sempre na qualidade da assistência prestada aos pacientes e ao correto preenchimento da guia e prontuário que são a principal demonstração disso. Visto a quantidade de instituições conveniadas e o grande montante de contas reunidas para serem auditadas, é de extrema importância que estas contas estejam com todos os dados e contendo todas as informações pertinentes aos tratamentos realizados.
\end{abstract}

Palavras- chave: Atendimento, Glosas, Conveniados, Policia Militar.

\section{Auditing in medical bills at the Hospital of the Military Police of Pernambuco}

\begin{abstract}
Currently, to remain competitive in the market, health institutions have to learn how associate low cost with excellent quality for their patients. The nursing audit represents the control function of the administrative process, making sure that care outcomes are consistent with the goals. Thus this study aimed at assessing the total value of monthly bills, major hospitals under the HPMPE and key appointments made with: outpatient visits, admissions and examinations. The survey was conducted in the field of medical accounts, which we assessed the bills received for audit in this hospital in August of 2010 units of capital and the interior. The search was guided by an accredited institution, amount billed, glossed value, amount paid and the reason for the disallowance total number of audited accounts. During this period, the quantification of a grand total bills paid $\mathrm{R} \$ 1,483,474.02$ in the capital and interior of Pernambuco state, this has generated a gloss of $\mathrm{R} \$$ $63,826.87$. So that we can decrease the percentage of total losses that nurses and hospital need to assess how institutions are being held nursing records, taking a critical view of its performance and willful, as we always carry out activities focusing on the quality of care provided to patients and correct annotation guide and handbook with is a major demonstration of that. Since the number of accredited institutions and the large amount of collected accounts to be audited, it is extremely important that these accounts are in good condition and contains all information pertinent to the treatments.
\end{abstract}

Keywords: Attendance, Glosses, covenants, Military Police

\footnotetext{
${ }^{1}$ Enfermeira, Especialista em Educação em Saúde UFPE- 2003 e Especialista em Auditoria de Saúde CEFAPP.

E-mail: gracecintra@hotmail.com.

${ }^{2}$ Zootecnista, Doutora em Nutrição Animal. E-mail: carlaxlsouza@yahoo.com.br;

${ }^{2}$ Enfermeira, Especialista em Auditoria de Saúde - CEFAPP 2011. E-mail: martadeu@ oi.com.br
} 


\section{Introdução}

A auditoria em Serviços de Saúde é um tema dos mais relevantes às Instituições de Saúde, porquanto essencial à manutenção da própria "saúde financeira" de tais organizações (MAIA e PAES, 2005). Deste modo, para manterem-se no mercado de forma competitiva, as instituições de saúde têm que aprender a associar baixos custos com excelência de qualidade para os seus pacientes. Conseqüentemente, as instituições de saúde têm sido compelidas a se organizarem como empresa desenvolvendo visão de negócio para sobreviverem a estas mudanças no mercado. Esta tendência mundial tem exigido dos profissionais envolvidos no setor habilidade na análise de custos para a prestação de serviços de saúde (BICHANGA, 2000).

A auditoria de enfermagem incorporou-se à rotina das instituições de saúde com o intuito de avaliar os aspectos qualitativos da assistência requerida pelo paciente, os processos internos e as contas hospitalares (SCARPARO et al., 2009).

A auditoria de enfermagem representa a função de controle do processo administrativo, verificando se os resultados da assistência estão de acordo com os objetivos (SILVA et al., 2009). Dessa forma, vem sendo concebida como o exame oficial dos registros de enfermagem com o objetivo de avaliar, verificar e melhorar a assistência, podendo concentrar-se nos registros e anotações de enfermagem (LOPES, 1998).

A auditoria tem surgido como uma ferramenta importante para mensuração da qualidade (Auditoria de cuidados) e custos (Auditoria de custos) das instituições de saúde. O processo de auditoria é conceituado como uma avaliação sistemática e formal de uma atividade realizada por pessoas não envolvidas diretamente em sua execução a fim de se determinar se a atividade está de acordo com os objetivos propostos GOTO, 2001). Desta forma, é possível evidenciar deficiências nas atividades desenvolvidas e apontar alternativas preventivas e corretivas para as mesmas (FARACO e ALBUQUERQUE, 2004).

A anotação de enfermagem é o registro do paciente durante as horas que ele permanece no ambiente hospitalar. O enfermeiro, que é um educador na sua essência, sendo parte responsável dos resultados obtidos por sua equipe, deve sensibilizar os membros dessa equipe quanto à importância da anotação e do envolvimento no processo de trabalho, podendo utilizar ferramentas educativas para tal, enfatizando que esta servirá como respaldo legal sobre a qualidade da assistência prestada ao cliente e quanto aos valores que a instituição receberá ou não dependendo desse registro (CFM, 2007).

D`Innocenzo (2010), Relata que nela podemos encontrar informações sobre sua saúde geral e dados administrativos. Deste modo, a anotação de enfermagem ou prontuário médico, é o meio pelo qual a informação da assistência prestada ao cliente pela a equipe multidisciplinar é transcrita e 
documentada, onde, serve de instrumento para auditoria, ajudando assim na auditoria das glosas hospitalares (D'INNOCENZO et al., 2010).

Este tipo de anotação, independentemente da filosofa e das teorias de enfermagem adotadas para fundamentar o processo de enfermagem na instituição, deve ser valorizada, uma vez que um dos meios para se avaliar os cuidados prestados ao cliente é a partir da adoção de indicadores de qualidade, para mensurar tanto o processo como os resultados da assistência de enfermagem (D`INNOCENZO et al., 2010).

Contudo, há contínuos problemas de glosas de contas médicas por operadoras de saúde, em convênios com instituições de saúde, as quais exigem que as contas estejam preenchidas corretamente, com registros claros e objetivos no prontuário tanto da evolução médica quanto da anotação de enfermagem, constando todas as condutas e procedimentos realizados para a garantia de pagamento (CAMELO e SILVA JUNIOR, 2005).

Maia e Paes (2005), discorrem em Manual de Auditoria de contas Médicas do Hospital Geral de Juiz de Fora, regras pertinentes a Auditorias especificamente em Hospitais Militares onde relatam que os auditores militares devem, impositivamente, para o êxito pleno de seus misteres, atuar em três níveis, ou, em outras palavras, em três momentos.

O primeiro deles, anterior à realização do ato médico, é o que pertence à análise, por uma Comissão de Comprovação de Urgências e Análise de Procedimentos de Alto Custo, da pertinência do procedimento médico solicitado.

O segundo nível de auditoria relatado por Maia e Paes (2005) é aquele que ocorre durante a realização do ato médico, consistindo na visita diária, realizada por membros da equipe de auditoria, às OCS que tenham pacientes do FUSEX internados em suas dependências. Nestas oportunidades, os auditores devem, sempre que possível, interagir com os pacientes e analisar a documentação nosológica dos mesmos (prontuários, exames complementares, condutas adotadas à diagnose e à terapêutica, dentre outras). Tais expedientes são essenciais ao êxito pleno do processo de auditoria, além de sinalizarem à direção e ao corpo clínico da OCS conveniada que está sendo feito um rigoroso acompanhamento dos trabalhos técnico profissionais e uma análise criteriosa e justa das contas apresentadas.

Para este segundo nível de auditoria é essencial que a equipe de auditores, além dos oficiais médicos, seja também integrada por profissional de nível superior, da área de enfermagem, preferencialmente com curso de especialização em auditoria de contas hospitalares.

O terceiro e último momento da auditoria é o referente à lisura técnica e contábil das contas que, certamente, só produzirá resultados proveitosos se integrado aos dois primeiros, anteriormente descritos. É irrealizável e improdutiva a lisura feita em faturas e/ou notas fiscais, sem um conhecimento mais aprofundado do caso clínico do paciente e sem uma acurada análise do seu prontuário médico (MAIA e PAES, 2005). 


\section{Metodologia}

O presente estudo não se caracteriza como pesquisa com seres humanos, utilizando os critérios padrão da resolução CNS 196/96, portanto não houve necessidade formal de submetê-lo a um comitê de ética e pesquisa.

Trata-se de um estudo de abordagem quantitativa e descritiva de campo transversal e exploratória no setor de contas médicas do Hospital da Polícia Militar do Recife.

A pesquisa foi realizada no setor de Contas médicas, onde avaliou-se as contas recebidas para auditoria no referido hospital no mês de agosto de 2010 pelas unidades da capital e do interior. O critério de pesquisa pautou-se pela instituição credenciada, valor cobrado, valor glosado, valor pago, motivo da glosa e o número total de contas auditadas.

\section{Resultados e Discussão}

Com o intuito de identificar o valor das contas auditadas e glosadas pelo Hospital da Polícia Militar de Pernambuco, na capital e no interior, durante o mês de Agosto do ano de 2010 e evidenciar as principais clínicas credenciadas, tipos de exames realizados, atendimentos ambulatoriais e internamentos, apresentamos os resultados nas Tabelas 1, 2 e 3, ao longo da discussão apresentada a seguir.

Durante este período quantificou-se um total de $\mathrm{R} \$ 1.223 .567,02$ de contas pagas na capital de Pernambuco, deste foi gerada uma glosa de $\mathrm{R} \$ 52.807,35$. Deste valor total é possível observar que neste período, apenas na capital Pernambucana, foram auditadas 2029 contas de atendimentos ambulatoriais, 1533 contas de exames por imagem, 117 contas de internamentos e 186 contas de exames laboratoriais, deste modo organizamos os resultados em tabelas de forma a descrever os principais clínicas credenciadas com o HPMPE, tipos de exames, atendimentos ambulatoriais e internamentos, e as eventuais glosas ocorridas com seus motivos conforme pode ser visto na Tabela 1.

Ferrreira et al.,(2009) realizaram uma pesquisa no serviço de urologia de um hospital privado de médio porte de Niterói, no Rio de Janeiro, tendo como referência os meses de outubro e novembro de 2007, eles encontraram neste período um total de 215 internações e 926 atendimentos ambulatoriais e perceberam que destes, 125 atendimentos foram glosados, onde geraram em total 4.380 itens glosados.

Apesar de não termos priorizado neste estudo a quantidade de itens glosados e sim seu valor gerado e o motivo da glosa, é possível observar que o fluxo de atendimentos neste estudo é maior que o observado por Ferreira et al., ${ }^{11}$ e apesar de o foco do estudo de Ferreira ser diferente do nosso é 
Id on Line Revista Multidisciplinar e de Psicologia

Id on Line Multidisciplinary and Psychology Journal

interessante salientar que o fluxo de atendimento ambulatorial é muitas vezes superior aos outros itens citados, como internamentos ou exames laboratoriais por exemplo. Além disso, é possível perceber também que a quantidade de itens glosados quando reunidos formam um importante montante, seja quantificado em itens ou valor cobrado, fato que demonstra a importância da auditoria nas contas médicas e quanto que a falta dela pode gerar em perdas para o hospital.

A questão de custos na área de saúde tem exigido profissionais cada vez mais qualificados que cooperem no resultado econômico da instituição. Dado que as perdas de materiais e medicamentos, as principais fontes lucrativas do hospital, são pouco controladas, a auditoria em enfermagem pode desempenhar um trabalho proativo em semelhança a este aspecto (FERREIRA et al., 2009).

Tabela 1 - Levantamento de contas auditadas do Hospital da PMPE na capital no mês de agosto de 2010

\begin{tabular}{|c|c|c|c|c|c|}
\hline Credenciado & $\begin{array}{l}\text { Valor } \\
\text { Cobrado }\end{array}$ & $\begin{array}{l}\text { Valor } \\
\text { Glosado }\end{array}$ & $\begin{array}{l}\text { Valor } \\
\text { Pago }\end{array}$ & Motivo da Glosa & $\mathrm{N}^{\mathrm{o}}$ Total de Contas Auditadas \\
\hline $\begin{array}{l}\text { ALEXANDRE } \\
\text { MACAHADO }\end{array}$ & 0 & 0 & 0 & - & Não houve atend. este mês \\
\hline ANGIO-RAD & 0 & 0 & 0 & - & Não houve atend. este mês \\
\hline BIOIMAGEM & 0 & 0 & 0 & - & Não houve atend. este mês \\
\hline CALHEIROS SIMÕES & 529,77 & 0 & 529,77 & - & 12 Atend. Ambulatoriais \\
\hline CEFIESPE & $2.478,54$ & 0 & $2.478,54$ & - & $\begin{array}{c}\text { 03 Atend. Ambulatoriais } \\
\text { (Fisioterapia, Fono, TO } \\
\text { Pedagogia) }\end{array}$ \\
\hline CEMOF & $1.714,79$ & 0 & $1.714,79$ & - & 39 Atend. Ambulatoriais \\
\hline CEMUPE & $2.219,98$ & 0 & $2.219,98$ & - & 06 Exames por imagem \\
\hline CEMUR & 0 & 0 & 0 & - & Não houve atend. este mês \\
\hline $\begin{array}{l}\text { CEN. ORTO. FISIO. } \\
\text { OLINDA } \\
\end{array}$ & 0 & 0 & 0 & - & Não houve atend. este mês \\
\hline $\begin{array}{l}\text { CENTRO MÉDICO E } \\
\text { IMUNOLÓGICO DE PE }\end{array}$ & 0 & 0 & 0 & - & Não houve atend. este mês \\
\hline $\begin{array}{l}\text { CENTRO MÉDICO } \\
\text { ATUAL PE }\end{array}$ & 0 & 0 & 0 & - & Não houve atend. este mês \\
\hline $\begin{array}{l}\text { CENTRO PE DE } \\
\text { ONCOLOGIA }\end{array}$ & $30.669,44$ & $4.876,80$ & $25.792,64$ & $\begin{array}{c}\text { Ausência dos descontos } 18 \% \text { sob os } \\
\text { quimioterápicos }\end{array}$ & 12 Atend. Ambulatoriais \\
\hline $\begin{array}{l}\text { CENTRO SUVAG } \\
\text { (OLINDA) } \\
\end{array}$ & 969,60 & 0 & 969,60 & - & 02 Atend. Ambulatoriais \\
\hline CENTRO SUVAG PE & $2.004,00$ & 0 & $2.004,00$ & - & 09 Atend Ambulatoriais \\
\hline CER & $1.122,39$ & 0 & $1.122,39$ & - & 13 Atend. Ambulatoriais \\
\hline CERPE & 0 & 0 & 0 & - & Não houve atend. este mês \\
\hline CICLO & 614,40 & 0 & 614,40 & - & 143 Atend. Ambulatoriais \\
\hline CLIFOR & 0 & 0 & 0 & - & Não houve atend. este mês \\
\hline $\begin{array}{l}\text { CLÍN. DERMAT. DO } \\
\text { DERBY }\end{array}$ & $4.959,36$ & 0 & $4.959,36$ & - & 164 Atend. Ambulatoriais \\
\hline $\begin{array}{l}\text { CLINICA DE OLHOS } \\
\text { HARLLEY STREET }\end{array}$ & 0 & 0 & 0 & - & Não houve atend. este mês \\
\hline CLINICA DE PULMÕES & 942,88 & 0 & 942,88 & - & $\begin{array}{l}11 \text { Exam. Ambulatoriais } \\
09 \text { Atend. Ambulatoriais }\end{array}$ \\
\hline CLINICA PAULO SERPA & 898,74 & 0 & 898,74 & - & 16 Atend. Ambulatoriais \\
\hline COOMEB & $15.758,04$ & 56,34 & $15.701,70$ & $\begin{array}{c}\text { Guia xerocada, duplicidade e } \\
\text { ausência }\end{array}$ & 532 Atend. Ambulatoriais \\
\hline
\end{tabular}


Id on Line Revista Multidisciplinar e de Psicologia

Id on Line Multidisciplinary and Psychology Journal

\begin{tabular}{|c|c|c|c|c|c|}
\hline COOPANEST & $29.367,89$ & 116,56 & $29.251,33$ & $\begin{array}{c}\text { Cobrança de } 04 \text { vasos (pagamos até } \\
03 \text { vasos) }\end{array}$ & 132 Honorários médicos \\
\hline COOPERCÁRDIO & 0 & 0 & 0 & - & Não houve atend. este mês \\
\hline COOPFÍSIO & $2.744,10$ & 0 & $2.744,10$ & - & 16 Atend. Ambulatoriais \\
\hline COPE & $6.951,01$ & 0 & $6.951,01$ & - & $\begin{array}{l}49 \text { Atend. Ambulatoriais } \\
\text { 05 Internamentos }\end{array}$ \\
\hline CRIO & $1.705,00$ & 0 & $1.705,00$ & - & 13 Atend. Ambulatoriais \\
\hline CTR & 0 & 0 & 0 & - & Não houve atend. este mês \\
\hline DENISE ALCÂNTARA & 0 & 0 & 0 & - & Não houve atend. este mês \\
\hline DERBIMAGEM & $6.890,41$ & 0 & $6.890,41$ & - & 79 Exames por Imagem \\
\hline DIAGMAX & 158,20 & 0 & 158,20 & - & 02 Exames por Imagem \\
\hline DIAGNO SÃO MARCOS & $10,688,01$ & 0 & $10,688,01$ & - & 70 Exames por Imagem \\
\hline $\begin{array}{l}\text { DIAGNÓSTICO } \\
\text { CARDÍACO }\end{array}$ & $9.402,70$ & 0 & $9.402,70$ & - & $\begin{array}{l}22 \text { Exames ambulatoriais } \\
53 \text { Exames p/ imagem }\end{array}$ \\
\hline DIAGNÓSTIKA & $1.200,00$ & 0 & $1.200,00$ & - & 06 Exames por Imagem \\
\hline DIGEST & 304,86 & 0 & 304,86 & - & 02 Exam. Ambulatoriais \\
\hline DILAB & $29.977,79$ & 0 & $29.977,79$ & - & 42 Exames por Imagem \\
\hline ENDOGASTRO & 0 & 0 & 0 & - & Não houve atend. este mês \\
\hline EXITO & $1.350,00$ & 378 & 972,00 & Sem Guia & 05 Honorários médicos \\
\hline FISIOARTE.COM & 871,20 & 0 & 871,20 & - & 04 Atend. Ambulatoriais \\
\hline FISIOCENTRO & 0 & 0 & 0 & - & Não houve atend. este mês \\
\hline FISIOCLIN & $1.532,10$ & 0 & $1.532,10$ & - & 17 Atend. Ambulatoriais \\
\hline FISIOLINDA & $2.144,62$ & 0 & $2.144,62$ & - & 19 Atend. Ambulatoriais \\
\hline FISIOMAX & $4.268,35$ & 0 & $4.268,35$ & - & 32 Atend. Ambulatoriais \\
\hline FISIOSAUDE & 108,90 & 0 & 108,90 & - & 01 Atend. Ambulatoriais \\
\hline GASTROLINDA & 0 & 0 & 0 & - & Não houve atend. este mês \\
\hline $\begin{array}{l}\text { GRUPO DE OFTAL. DE } \\
\text { PE }\end{array}$ & 0 & 0 & 0 & - & Não houve atend. este mês \\
\hline HOPE & - & 0 & & - & Não houve atend. este mês \\
\hline HORE & $71.793,79$ & $2.185,4$ & $69.603,39$ & $\begin{array}{c}\text { Pacote diferente + Excesso de } \\
\text { descartáveis + taxa do CC } \\
\text { diferentes da tabela, sem guia } \\
\text { valores diferentes das tabelas: } \\
\text { brasindice e PMPE } \\
\end{array}$ & $\begin{array}{l}01 \text { Exam. Ambulatorial } \\
524 \text { Atend. Ambulatoriais } \\
21 \text { Internamentos }\end{array}$ \\
\hline HOSPITAL ALFA & $1.761,94$ & 0 & $1.761,94$ & $\begin{array}{c}\text { Quant. Exec. Mat/ med diferente da } \\
\text { tabela da PM }\end{array}$ & 02 Exames por Imagem \\
\hline HOSPITAL D'AVILA & & 0 & & & Não houve atend. este mês \\
\hline $\begin{array}{l}\text { HOSPITAL DE OLHOS } \\
\text { SANTA LUZIA }\end{array}$ & $33.242,79$ & 10,8 & $33.232,71$ & Valor da consulta diferente & 63 Atend. Ambulatoriais \\
\hline HOSPITAL ESPERANÇA & $10.742,06$ & 283,56 & $10.503,50$ & $\begin{array}{l}\text { Quant. exec. material médico em } \\
\text { bloco cirúrgico }\end{array}$ & $\begin{array}{l}04 \text { Atend. Ambulatoriais } \\
13 \text { Exames por Imagem } \\
01 \text { Internamento }\end{array}$ \\
\hline $\begin{array}{l}\text { HOSPITAL JAYME DA } \\
\text { FONTE }\end{array}$ & $345.776,7$ & $9.416,4$ & $336.360,3$ & $\begin{array}{l}\text { Honorários de fisioterapia, quant. } \\
\text { de mat/ med. (sangoflex) }\end{array}$ & $\begin{array}{l}2 \text { Atend. Ambulatoriais } \\
04 \text { Exam. Diversos } \\
47 \text { Intern. Hospitalar }\end{array}$ \\
\hline IMUNOPAT & $1.012,80$ & 0 & $1.012,80$ & - & 04 Atend. Ambulatoriais \\
\hline $\begin{array}{l}\text { INSTITUTO DE VISÃO } \\
\text { DO RECIFE }\end{array}$ & - & 0 & - & - & Não houve atend. este mês \\
\hline $\begin{array}{l}\text { INT. FISIO. DUARTE } \\
\text { COELHO }\end{array}$ & - & 0 & - & - & Não houve atend. este mês \\
\hline IVO ROESLER & $31.842,39$ & 0 & $31.842,39$ & - & 17 Atend. Ambulatoriais \\
\hline
\end{tabular}


Id on Line Revista Multidisciplinar e de Psicologia

Id on Line Multidisciplinary and Psychology Journal

\begin{tabular}{|c|c|c|c|c|c|}
\hline $\begin{array}{l}\text { LAB. ADONES } \\
\text { CARVALHO }\end{array}$ & $6.202,75$ & 0 & $6.202,75$ & - & 57 Exames Laboratoriais \\
\hline $\begin{array}{l}\text { LAB. FERNANDO } \\
\text { TRAVASSOS }\end{array}$ & - & 0 & - & - & Não houve atend. este mês \\
\hline $\begin{array}{l}\text { LABORATÓRIO GILSON } \\
\text { CIDRIN }\end{array}$ & $5.984,89$ & 271,9 & $5.676,99$ & $\begin{array}{c}\text { Valores Diferentes da tab do } \\
\text { HPMPE }\end{array}$ & 100 Exam. Laboratoriais \\
\hline LIAC & - & 0 & - & - & Não houve atend. este mês \\
\hline MAXICLINICAS & - & 0 & - & - & Não houve atend. este mês \\
\hline MEMORIAL OFTALMO & - & 0 & - & - & Não houve atend. este mês \\
\hline MULTIHEMO & $310.221,8$ & $6.994,2$ & $303.227,6$ & Mat/ med diferente tabela Gremes & $\begin{array}{l}43 \text { Atend. Ambulatoriais } \\
47 \text { Atend. Ambulatoriais }\end{array}$ \\
\hline MULTIMAGEM & $69.038,16$ & 0 & $69.038,16$ & - & 260 Exames por imagem \\
\hline MULTIRIM & - & 0 & - & - & Não houve atend. este mês \\
\hline $\mathrm{NEOH}$ & $25.350,23$ & 0 & $25.350,23$ & - & 07 Atend. Ambulatoriais \\
\hline $\begin{array}{l}\text { NEUROFISIOLOGIA } \\
\text { CLINICA }\end{array}$ & $5.013,36$ & 0 & $5.013,36$ & - & 30 Atend. Ambulatoriais \\
\hline $\begin{array}{l}\text { OFICINA TERA. EDU. } \\
\text { ESPECIAL }\end{array}$ & $2.544,00$ & 0 & $2.544,00$ & - & 02 Atend. Ambulatoriais \\
\hline $\begin{array}{l}\text { ORGANIZAÇÃO DE } \\
\text { HOSPITAIS DE PE }\end{array}$ & $3.155,80$ & 0 & $3.155,80$ & - & 2 Intern. Hospitalar \\
\hline ORTOCENTRO & $1.470,12$ & 0 & $1.470,12$ & Ausência de descrição cirúrgica & $\begin{array}{l}06 \text { Atend. Ambulatoriais } \\
02 \text { Honorários médicos }\end{array}$ \\
\hline ORTOFACE RECIFE & $7.323,93$ & $1.368,5$ & $5.955,40$ & $\begin{array}{c}\text { Honorários médicos sob cirurgia na } \\
\text { mesma cavidade }\end{array}$ & 13 Honorários médicos \\
\hline PASM & 142,84 & 0 & 142,84 & - & 08 Exames Laboratoriais \\
\hline PATLAB & 987,48 & 0 & 987,48 & - & 21 Exames Laboratoriais \\
\hline PREVENCOR & $12.543,84$ & 0 & $12.543,84$ & - & 288 Atend. Ambulatoriais \\
\hline PREVIMAGEM & 104,9 & 0 & 104,9 & - & 2 Exames $\mathrm{p} /$ imagem \\
\hline PRO- NEURO & 284,1 & 0 & 284,1 & - & 04 Exam Ambulatoriais \\
\hline PROCÁRDIO & $10.666,55$ & 49,68 & $10.616,87$ & Cobranças indevidas & 132 Exames por Imagem \\
\hline PRONTIMAGEM & $32.956,94$ & 65,78 & $32.891,16$ & Sem guia & $\begin{array}{c}5 \text { Internamentos } \\
26 \text { Atend. Ambulatoriais }\end{array}$ \\
\hline $\begin{array}{l}\text { PRONTO SOCORRO } \\
\text { INFANTIL JORGE DE } \\
\text { MEDIEROS }\end{array}$ & $24.444,29$ & $5.458,7$ & $18.985,59$ & $\begin{array}{l}\text { Cobranças indevidas de: tx de enf., } \\
\text { de visita hospitalar, monitor, } \\
\text { aspirador, BIC e oxímetro incluso } \\
\text { na diária de UTI, honorários da } \\
\text { uteista e fisioterapeuta, quant. Exc. } \\
\text { preço de mat/ med, valor de raio X }\end{array}$ & 04 Intern. Hospitalar \\
\hline PROTOLINDA & 0 & 0 & 0 & - & Não houve atend. este mês \\
\hline QUALIMAGEM & $10.188,54$ & 0 & $10.188,54$ & - & 136 Exame por Imagem \\
\hline RADIOFACE & 0 & 0 & 0 & - & Não houve atend. este mês \\
\hline RENAL SERVICES & 0 & 0 & 0 & - & Não houve atend. este mês \\
\hline RESPIRAR & 0 & 0 & 0 & - & Não houve atend. este mês \\
\hline SALFISIO & $3.864,50$ & 0 & $3.864,50$ & - & 19 Atend. Ambulatoriais \\
\hline $\begin{array}{c}\text { SANTAORIO } \\
\text { PSIQUIATRICO DO } \\
\text { RECIFE } \\
\end{array}$ & 0 & 0 & 0 & - & Não houve atend. este mês \\
\hline $\begin{array}{l}\text { SANATORIO PSI. } \\
\text { RECUPERAÇÃO }\end{array}$ & $16.947,7$ & 0 & $16.947,70$ & - & 16 Intern. Hospitalar \\
\hline SANLIFE & 0 & 0 & 0 & - & Não houve atend. este mês \\
\hline SECOR & 0 & 0 & 0 & - & Não houve atend. este mês \\
\hline SEQUIPE & $3.761,44$ & 0 & $3.761,44$ & - & 07 Atend. Ambulatoriais \\
\hline
\end{tabular}


Id on Line Revista Multidisciplinar e de Psicologia

Id on Line Multidisciplinary and Psychology Journal

\begin{tabular}{|c|c|c|c|c|c|}
\hline SOS MÃO & 783,00 & 4,57 & 778,43 & Material em curativo & $\begin{array}{l}13 \text { Atend. Ambulatoriais } \\
02 \text { Exames por Imagem }\end{array}$ \\
\hline $\begin{array}{c}\text { TERAPIA E } \\
\text { MOVIMENTO }\end{array}$ & 603,80 & 0 & 603,80 & - & 08 Atend. Ambulatoriais \\
\hline TOPMAGEM & 0 & 0 & 0 & - & Não houve atend. este mês \\
\hline UDO DERBY & $47.101,53$ & 0 & $47.101,53$ & - & 450 Exames por Imagem \\
\hline $\begin{array}{c}\text { UNIDADE DE VÍDEO } \\
\text { ENDOSCOPIA } \\
\text { DIGESTIVA }\end{array}$ & $4.100,00$ & 0 & $4.100,00$ & - & 02 Exames por Imagem \\
\hline UNIMAGEM & 0 & 0 & 0 & & Não houve atend. este mês \\
\hline ININEURO RECIFE & $46.405,23$ & $4.835,4$ & $41.569,88$ & $\begin{array}{l}\text { Valores cobrados diferentes da } \\
\text { tabela PM + hora extra indevida }\end{array}$ & 98 Exame por Imagem \\
\hline UNITOMO & 0 & 0 & 0 & - & Não houve atend. este mês \\
\hline URO & 497,00 & 0 & 497,00 & - & 03 Atend. Ambulatoriais \\
\hline $\begin{array}{l}\text { TOTAL GERAL POR } \\
\text { ESPECIFICAÇÃO DAS } \\
\text { CONTAS AUDITADAS } \\
\text { NA CAPITAL }\end{array}$ & $\mathbf{0}$ & $\mathbf{0}$ & $\mathbf{0}$ & - & $\begin{array}{l}295 \text { Honorários médicos } \\
2029 \text { Atend. ambulatoriais } \\
117 \text { Internamentos } \\
1533 \text { Exames p imagem } \\
186 \text { Ex. laboratoriais } \\
186 \text { Exames diversos }\end{array}$ \\
\hline $\begin{array}{c}\text { TOTAL GERAL EM } \\
\text { VALORES CAPITAL }(\mathrm{R} \$)\end{array}$ & $1.276,374$ & $\mathbf{5 2 . 8 0 7}$ & $1.233,567$ & - & - \\
\hline
\end{tabular}

Se analisarmos as duas tabelas desta pesquisa (Tabela $1 \mathrm{e}$ Tabela 2), referentes às contas dos hospitais conveniados ao hospital da PMPE da capital e interior do estado durante um mês, perceberemos o grande fluxo de instituições conveniadas em atendimento, sendo 103 instituições na capital e 108 no interior, destas, $17,4 \%$ e $27 \%$ tiveram suas contas glosadas respectivamente.

Os motivos das glosas foram diversos, porém percebeu-se que, a falta de guia, a cobrança indevida de serviços ou taxas e cobrança diferente da tabela da PM ocorreram diversas vezes. Já Ferreira et al.,(2009) encontrou em sua pesquisa que a maioria de suas glosas (53\%) foram referentes ao item medicamentos.

As contas geradas pelos nossos credenciados do interior podem ser vistas na Tabela 2, só as contas glosadas geraram um total de $\mathrm{R} \$ 11.019,52$, e um total geral de contas de $\mathrm{R} \$ 259.906,79$, estas representaram um total de 2461 Contas de atendimentos ambulatoriais, 712 Contas de exames por Imagem, 200 contas de exames diversos, 708 contas de exames laboratoriais e 249 contas de internamento.

Tabela 2 - Levantamento de contas auditadas do Hospital da PMPE no Interior no mês de agosto de 2010

\begin{tabular}{|c|c|c|c|c|c|}
\hline Credenciado & $\begin{array}{c}\text { Valor } \\
\text { Cobrado }\end{array}$ & $\begin{array}{c}\text { Valor } \\
\text { Glosado }\end{array}$ & $\begin{array}{l}\text { Valor } \\
\text { Pago }\end{array}$ & Motivo da Glosa & $\begin{array}{c}\mathrm{N}^{\mathbf{0}} \text { Total de Contas } \\
\text { Auditadas }\end{array}$ \\
\hline \multicolumn{6}{|l|}{$\begin{array}{c}2^{\circ} \text { BPM (Nazaré da } \\
\text { Mata) }\end{array}$} \\
\hline $\begin{array}{c}\text { CLINICA DIOCLÉCIO } \\
\text { COUTINHO }\end{array}$ & $7.825,53$ & 0 & $7.825,53$ & - & $\begin{array}{l}56 \text { Atend. Ambulatoriais } \\
55 \text { Exames por imagem } \\
29 \text { Atend. Laboratoriais } \\
07 \text { Exames ambulatoriais }\end{array}$ \\
\hline $\begin{array}{l}\text { CLÍNICA DE OLHOS DE } \\
\text { CARPINA }\end{array}$ & 672,00 & 0 & 672,00 & - & 20 Atend. Ambulatoriais \\
\hline
\end{tabular}


Id on Line Revista Multidisciplinar e de Psicologia

Id on Line Multidisciplinary and Psychology Journal

\begin{tabular}{|c|c|c|c|c|c|}
\hline $\begin{array}{l}\text { CENTRO SAU. REAB. } \\
\text { PAUDALHO } \\
\end{array}$ & 588,3 & 37,5 & 550,8 & sem laudo & 07 Atend. Ambulatoriais \\
\hline CLINICOR & $1.037,55$ & 0 & 1037,55 & - & $\begin{array}{l}16 \text { Atend. Ambulatoriais } \\
02 \text { exames por imagem }\end{array}$ \\
\hline $\begin{array}{l}\text { MEMORIAL HOSPITAL } \\
\text { DE GOIANA }\end{array}$ & 0 & 0 & 0 & - & Não houve atend. este mês \\
\hline \multicolumn{6}{|l|}{$3^{\circ}$ BPM (Arco Verde) } \\
\hline $\begin{array}{l}\text { HOSPITAL MEMORIAL } \\
\text { ARCOVERDE }\end{array}$ & $19.676,16$ & 84 & $19.592,2$ & $\begin{array}{l}\text { Cobranças indevidas de taxas e } \\
\text { materiais médicos }\end{array}$ & $\begin{array}{l}264 \text { Atend Ambulatoriais } \\
07 \text { Exames por imagem } \\
\text { 07 Internamentos } \\
22 \text { Exames Laboratoriais }\end{array}$ \\
\hline $\begin{array}{c}\text { CLIN. REUM, FISIO, E } \\
\text { REABILITAÇÃO } \\
\text { ARCOVERDE }\end{array}$ & 357,10 & 0 & 357,10 & - & 03 Atend. Ambulatoriais \\
\hline ARCOLAB & 703,68 & 0 & 703,68 & - & 16 Exames laboratoriais \\
\hline $\begin{array}{l}\text { ARCOVERDE DIAG. } \\
\text { MÉDICO }\end{array}$ & 0 & 0 & 0 & - & Não houve atend. este mês \\
\hline FISIOCENTER & $1.546,90$ & 0 & $1.546,90$ & - & 08 Atend. ambulatoriais \\
\hline SEMI & $2.328,24$ & 0 & $2.328,24$ & - & 55 Exames laboratoriais \\
\hline NEUROIMAGEM & $6.271,30$ & 128 & $6.143,30$ & $\begin{array}{l}\text { Ausência de laudo, desconformidade } \\
\text { com a tab. PMPE }\end{array}$ & $\begin{array}{l}20 \text { Atend. ambulatoriais } \\
129 \text { Exames por imagem }\end{array}$ \\
\hline ADIMED & 447,28 & 0 & 447,28 & - & 06 Exames laboratoriais \\
\hline \multicolumn{6}{|l|}{$4^{\circ}$ BPM (CARUARU) } \\
\hline CEFÍSIO & $1.769,50$ & 0 & $1.769,50$ & - & 12 Atend. ambulatoriais \\
\hline ENDIC & 480 & 0 & 480 & - & 04 Exames por imagem \\
\hline $\begin{array}{c}\text { CLÍNICA DE } \\
\text { ULTRASSONOGRAFIA } \\
\text { (Unimagem Caruaru) }\end{array}$ & $4.502,08$ & 0 & $4.502,08$ & - & 77 Exames por imagem \\
\hline $\begin{array}{c}\text { CENTRO DE } \\
\text { FONOAUDIOLOGIA DE } \\
\text { CARUARU } \\
\end{array}$ & 438 & 0 & 438 & & 05 Atend. ambulatoriais \\
\hline CFS & & 0 & & & Não houve atend. este mês \\
\hline LAAF & $6.215,68$ & 0 & $6.215,68$ & & 100 Exames laboratoriais \\
\hline CECOC & & & & & Não houve atend. este mês \\
\hline $\begin{array}{l}\text { CLÍNICA SÃO JUDAS } \\
\text { TADEU }\end{array}$ & 135 & 0 & 135 & & 01 Exames p/ imagem \\
\hline $\begin{array}{l}\text { HOSPITALJESUS } \\
\text { PEQUENINO } \\
\end{array}$ & 274,71 & 39,51 & 235,2 & Ausência de laudo, ECG & 10 Atend. ambulatoriais \\
\hline $\begin{array}{l}\text { INTITUTO DA VISÃO } \\
\text { DE PE }\end{array}$ & 559,78 & 0 & 559,78 & & 16 Atend. ambulatoriais \\
\hline ESPAÇO VIDA & 446,5 & 0 & 446,5 & & 07 Atend. ambulatoriais \\
\hline $\begin{array}{l}\text { CENTRO DE } \\
\text { ONCOLOGIA DE } \\
\text { CARUARU }\end{array}$ & 730,66 & 0 & 730,66 & & 01 Atend. ambulatoriais \\
\hline \multicolumn{6}{|l|}{$5^{\circ}$ BPM (Petrolina) } \\
\hline $\begin{array}{l}\text { CLINICA DE FISIO. E } \\
\text { OSTEOPATIA SÃO } \\
\text { MARCOS }\end{array}$ & 0 & 0 & 0 & - & Não houve atend. este mês \\
\hline CEDILA & $5.615,32$ & 0 & $5.615,32$ & - & 105 Ex. Laboratoriais \\
\hline CDI & $14.199,46$ & $1.981,93$ & $12.217,5$ & Cobrança de taxa indevida de oxímetro & 20 Exames por imagem \\
\hline NEUROCARDIO & $28.937,24$ & $2.960,24$ & $25,977,0$ & $\begin{array}{c}\text { Falta de aut. Mat. Especial + cobrança } \\
\text { indevida de omeprazol }+ \text { taxa de } \mathrm{O} 2 \mathrm{e} \\
\text { nebulização indevida }+ \text { taxa de medico } \\
\text { indevida }\end{array}$ & $\begin{array}{l}109 \text { Atend Ambulatoriais } \\
01 \text { Exames por imagem } \\
08 \text { Intern. Hospitalar }\end{array}$ \\
\hline
\end{tabular}


Id on Line Revista Multidisciplinar e de Psicologia

Id on Line Multidisciplinary and Psychology Journal

\begin{tabular}{|c|c|c|c|c|c|}
\hline $\begin{array}{l}\text { INST. DE OLHOS DO } \\
\text { VALE DO SÃO } \\
\text { FRANCISCO }\end{array}$ & $5.116,80$ & 791,51 & $4.325,29$ & $\begin{array}{l}\text { Solicitação de exame diferente do } \\
\text { realizado, preço diferente da tabela, } \\
\text { cobrança indevida de pacote }\end{array}$ & $\begin{array}{l}69 \text { Atendimentos } \\
\text { ambulatoriais }\end{array}$ \\
\hline SEMPE & $3.285,84$ & 0 & $3.285,84$ & - & 81 Atend. ambulatoriais \\
\hline $\begin{array}{l}\text { CLINICA UROLÓGICA } \\
\text { DE PETROLINA }\end{array}$ & $1.408,15$ & 67,76 & $1.340,39$ & Preços diferentes da tabela PM & 11 Atend. ambulatoriais \\
\hline VIP ENDOSCOPIA & 820 & 0 & 820 & & Não houve atend. este mês \\
\hline CECOG & $1.052,63$ & 226,36 & 826,27 & Preços diferentes da tabela PM & 08 Atend. ambulatoriais \\
\hline UROMED DAY & 481,07 & 122,99 & 358,58 & $\begin{array}{c}\text { Preços diferentes da tabela PM + } \\
\text { excesso de material }\end{array}$ & 05 Atend. Ambulatoriais \\
\hline HISTOTEC PATOLOGIA & 0 & 0 & 0 & - & Não houve atend. este mês \\
\hline $\begin{array}{l}\text { PRÓ-MATRE DO } \\
\text { JUAZEIRO }\end{array}$ & $6.727,66$ & 720,34 & $6.007,32$ & Sem fatura e laudo & $\begin{array}{l}70 \text { Atend. Ambulatoriais } \\
38 \text { Exames por imagem } \\
15 \text { Exames laboratoriais } \\
\end{array}$ \\
\hline CLIOFT & 0 & 0 & 0 & - & Não houve atend. este mês \\
\hline CARDIOLAB & 0 & 0 & 0 & - & Não houve atend. este mês \\
\hline $\begin{array}{l}\text { CLINICA MUCCINE } \\
\text { GUEDES } \\
\end{array}$ & $2.306,45$ & 118,44 & $2.188,01$ & Valores dos filmes diferente da tabela & 43 Exames por imagem \\
\hline \multicolumn{6}{|l|}{$7^{\circ}$ BPM (Ouricuri) } \\
\hline $\begin{array}{c}\text { HOSP. E } \\
\text { MATERNIDADE } \\
\text { SANTA MARIA }\end{array}$ & $3.017,02$ & 0 & $3.017,02$ & - & $\begin{array}{c}06 \text { Atend. Ambulatoriais } \\
07 \text { Exames laboratoriais } \\
03 \text { Intern. hospitalar }\end{array}$ \\
\hline $\begin{array}{l}\text { CLINICA DE } \\
\text { REABILITAÇÃO } \\
\text { ARARIPE } \\
\end{array}$ & 871,1 & 0 & 871,1 & - & 06 Atend. Ambulatoriais \\
\hline RADMAGEM & $2,428,28$ & 213,2 & $2.215,08$ & Preços diferentes da tabela & 21 Exames de imagem \\
\hline $\begin{array}{l}\text { CENTRO DE ANÁLISES } \\
\text { CLINICAS } \\
\text { ESPECIALIZADAS }\end{array}$ & 580,84 & 0 & 580,84 & - & 78 Exames laboratoriais \\
\hline $\begin{array}{l}\text { CLÍNICA MÉDICA DO } \\
\text { ARARIPE }\end{array}$ & 168,5 & 0 & 168,5 & - & $\begin{array}{l}02 \text { Atend. Ambulatoriais } \\
01 \text { Exame por imagem }\end{array}$ \\
\hline $\begin{array}{c}\text { CARDIOLOGIA } \\
\text { CONSULTÓRIOS } \\
\end{array}$ & 572,92 & 0 & 572,92 & - & 14 Atend. Ambulatoriais \\
\hline \multicolumn{6}{|l|}{$8^{\circ}$ BPM (Salgueiro) } \\
\hline $\begin{array}{c}\text { LABOTATÓRIO } \\
\text { SERTANEJO DE } \\
\text { ANÁLISES CLÍNICAS } \\
\end{array}$ & $1.764,96$ & 0 & $1.764,96$ & - & 36 Exames laboratoriais \\
\hline RADIODERME & $1.010,11$ & 0 & $1.010,11$ & - & $\begin{array}{l}12 \text { Atend. Ambulatoriais } \\
14 \text { Exame por imagem }\end{array}$ \\
\hline $\begin{array}{c}\text { CLINICA DE } \\
\text { FONOTERAPIA }\end{array}$ & 0 & 0 & 0 & - & Não houve atend. este mês \\
\hline $\begin{array}{l}\text { PRONTOSOCORRO } \\
\text { SÃO FRANCISCO }\end{array}$ & 0 & 0 & 0 & - & Não houve atend. este mês \\
\hline $\begin{array}{c}\text { CLINICA } \\
\text { ESPECIALIZADA } \\
\text { SERTÃO CENTRAL }\end{array}$ & $11.170,25$ & 544,01 & $10,626,2$ & $\begin{array}{l}\text { Cobrança de cons. como fisioterapia } \\
\text { duplicada de ficha, excesso de diária, } \\
\text { sem laudo, cobrança indevida }\end{array}$ & $\begin{array}{l}72 \text { Atend. Ambulatoriais } \\
28 \text { Exame por imagem } \\
12 \text { Intern. Hospitalares }\end{array}$ \\
\hline $\begin{array}{l}\text { PRONTOCLINICA SÃO } \\
\text { LUCAS }\end{array}$ & $3.020,44$ & 252,82 & $2,767,62$ & $\begin{array}{c}\text { Sem laudo + valor diferente da consulta } \\
\text { com psicólogo }\end{array}$ & $\begin{array}{l}\text { 41 Atend. Ambulatoriais } \\
06 \text { Exame por imagem } \\
02 \text { Exames laboratoriais }\end{array}$ \\
\hline $\begin{array}{c}\text { CLINICA DE } \\
\text { FISIOLOGIA SERTÃO } \\
\text { CENTRAL } \\
\end{array}$ & 0 & 0 & 0 & - & Não houve atend. este mês \\
\hline \multicolumn{6}{|l|}{$9^{\circ}$ BPM (Caruaru) } \\
\hline LAMEG & 441,47 & 0 & 441,47 & & 09 Exames laboratoriais \\
\hline $\begin{array}{l}\text { HOSPITAL MONTE } \\
\text { SINAI }\end{array}$ & $17.552,83$ & 339,3 & $17.231,5$ & $\begin{array}{l}\text { Cobranças indevidas de: equipo, tx sala } \\
\text { de obs, medicamento (omeprazol) }\end{array}$ & $\begin{array}{l}73 \text { Atend. Ambulatoriais } \\
08 \text { Internamentos } \\
\text { 05 Exames ambulatoriais }\end{array}$ \\
\hline
\end{tabular}


Id on Line Revista Multidisciplinar e de Psicologia

Id on Line Multidisciplinary and Psychology Journal

\begin{tabular}{|c|c|c|c|c|c|}
\hline $\begin{array}{l}\text { CASADE SAÚDE E } \\
\text { MATERNIDADE NSR } \\
\text { DO PERPETUO } \\
\text { SOCORRO }\end{array}$ & $35.078,57$ & 189,81 & $34.888,7$ & $\begin{array}{c}\text { Cobranças indevidas de taxa de } \\
\text { material médico }\end{array}$ & $\begin{array}{l}335 \text { Atend. Ambulatoriais } \\
70 \text { Exames de imagem } \\
46 \text { Exames diversos } \\
18 \text { Exames laboratoriais }\end{array}$ \\
\hline $\begin{array}{l}\text { CLINICA DE } \\
\text { ORTOPEDIA E } \\
\text { FRATURA } \\
\end{array}$ & $5.428,68$ & 563 & $4.865,68$ & Sem laudo e sem freqüência & $\begin{array}{l}310 \text { Atend. Ambulatoriais } \\
31 \text { exames por imagem }\end{array}$ \\
\hline DIAGNOS LABO & 0 & 0 & 0 & - & Não houve atend. este mês \\
\hline CARDIOLAB & $5.447,27$ & 0 & $5.447,27$ & - & $\begin{array}{l}47 \text { Atend. Ambulatoriais } \\
21 \text { Exames por imagem } \\
08 \text { Exames laboratoriais }\end{array}$ \\
\hline SEMOGA & $7.358,01$ & 0 & $7.358,01$ & - & 137 Exames ambulatórias \\
\hline LAGAM & & 0 & & - & Não houve atend. este mês \\
\hline $\begin{array}{c}\text { CENTR. DIAGNÓSTICO } \\
\text { Dr. LUIZ GABRIEL } \\
\end{array}$ & $3.443,55$ & 0 & $3.443,55$ & - & 53 Exames por imagem \\
\hline CLINICAL CENTER & 0 & 0 & 0 & - & Não houve atend. este mês \\
\hline LAB. ADOLFO LUTZ & $4.974,32$ & 58,2 & $4.916,12$ & Sem Laudo & 98 Exames laboratoriais \\
\hline \multicolumn{6}{|l|}{$10^{\circ}$ BPM (Palmares) } \\
\hline $\begin{array}{l}\text { CENTRO MÉDICO } \\
\text { SANTA PAULA }\end{array}$ & $2.676,12$ & 0 & $2.676,12$ & - & $\begin{array}{l}16 \text { Atend. Ambulatoriais } \\
14 \text { Exames de imagem } \\
03 \text { Exames diversos } \\
24 \text { Exames laboratoriais }\end{array}$ \\
\hline \multicolumn{6}{|l|}{$\begin{array}{l}4^{\circ} \text { BPM (Serra } \\
\text { Talhada) }\end{array}$} \\
\hline $\begin{array}{l}\text { CLIMATIN- CLINICA } \\
\text { MATERNO INFANTIL }\end{array}$ & $2.890,79$ & 0 & $2.890,79$ & - & $\begin{array}{l}14 \text { Atend. Ambulatoriais } \\
11 \text { Exames por imagem }\end{array}$ \\
\hline $\begin{array}{l}\text { CLINICA FRANCISCO } \\
\text { ANSELMO }\end{array}$ & $10.073,02$ & 113,28 & $9.959,74$ & $\begin{array}{c}\text { Valor USG diferente }+ \text { sem laudo }+ \\
\text { sem guia }\end{array}$ & $\begin{array}{l}173 \text { Atend Ambulatoriais } \\
15 \text { Exames de imagem } \\
04 \text { Intern. Hospitalar } \\
37 \text { Exames laboratoriais }\end{array}$ \\
\hline $\begin{array}{l}\text { CASA DE SAÚDE E } \\
\text { MATERNIDADE SÃO } \\
\text { VICENTE }\end{array}$ & $2.634,08$ & 64,21 & $2.569,87$ & Valores de material médico & $\begin{array}{l}32 \text { Atend. Ambulatoriais } \\
02 \text { Exames laboratoriais } \\
02 \text { Intern. Hospitalares }\end{array}$ \\
\hline $\begin{array}{c}\text { CLINICA DE FISIO. E } \\
\text { REABILITAÇÃO SERRA } \\
\text { TALHADA } \\
\end{array}$ & $2.288,90$ & 0 & $2.288,90$ & - & 10 Atend. Ambulatoriais \\
\hline $\begin{array}{l}\text { LABO. ADILENE } \\
\text { BARBOSA }\end{array}$ & $4.305,76$ & 59,68 & $4.246,08$ & Sem laudo & 78 Exames laboratoriais \\
\hline $\begin{array}{c}\text { CLINICA DE FONO S. } \\
\text { TALHADA (Maria do } \\
\text { Socorro) }\end{array}$ & $1.300,00$ & 0 & $1.300,00$ & - & 10 Atend. Ambulatoriais \\
\hline CLIFES & 448,2 & 0 & 448,2 & - & 04 Atend. Ambulatoriais \\
\hline CLINICA DA CRIANÇA & $4.113,82$ & 0 & $4.113,82$ & - & $\begin{array}{l}52 \text { Atend. Ambulatoriais } \\
03 \text { Intern Hospitalar }\end{array}$ \\
\hline $\begin{array}{l}\text { CLINICA DE } \\
\text { OTORRINO DE S. } \\
\text { TALHADA } \\
\end{array}$ & 417,21 & 33,6 & 383,61 & Sem guia & 12 Atend. Ambulatoriais \\
\hline CLINICARD & $2.811,80$ & 190,48 & $2.621,39$ & Sem guia at. + sem laudo & 30 Atend. Ambulatoriais \\
\hline $\mathrm{HGU}$ & 0 & 0 & 0 & - & Não houve atend. este mês \\
\hline SUST & 905,48 & 97,19 & 808,29 & Sem guia & 12 Exames por imagem \\
\hline \multicolumn{6}{|l|}{$15^{\circ}$ BPM (Belo Jardim) } \\
\hline $\begin{array}{l}\text { ROSICLEIDE BARBOSA } \\
\quad \text { (Clinica Santa Rita) }\end{array}$ & 0 & 0 & 0 & - & Não houve atend. este mês \\
\hline $\begin{array}{c}\text { LABORATÓRIO DE } \\
\text { ANÁLISES CLINICAS } \\
\text { MONSERRATE }\end{array}$ & 0 & 0 & 0 & - & Não houve atend. este mês \\
\hline $\begin{array}{c}\text { REABILITAR CLINICA } \\
\text { DE FISIO SOCIEDADE } \\
\text { SIMPLES }\end{array}$ & 0 & 0 & 0 & - & Não houve atend. este mês \\
\hline
\end{tabular}


Id on Line Revista Multidisciplinar e de Psicologia

Id on Line Multidisciplinary and Psychology Journal

\begin{tabular}{|c|c|c|c|c|c|}
\hline LABOPAC & 377,56 & 0 & 377,56 & - & 11 Exames Laboratoriais \\
\hline $\begin{array}{l}\text { CASA DE SAÚDE SÃO } \\
\text { JOSÉ }\end{array}$ & 0 & 0 & 0 & - & Não houve atend. este mês \\
\hline HIDRO FISIO & 0 & 0 & 0 & - & Não houve atend. este mês \\
\hline $\begin{array}{l}\text { CENTRO HOSPITAL DE } \\
\text { PESQUEIRA }\end{array}$ & $5.353,18$ & 193,17 & $5.160,01$ & Sem Laudo + sem frequência & $\begin{array}{l}51 \text { Atend. Ambulatoriais } \\
15 \text { Exames por imagem } \\
03 \text { Exames laboratoriais }\end{array}$ \\
\hline \multicolumn{6}{|l|}{$22^{\circ}$ BPM (Surubim) } \\
\hline $\begin{array}{l}\text { CLINICA DIOCLÉCIO } \\
\text { COUTINHO }\end{array}$ & $1.621,72$ & 0 & $1.621,72$ & - & $\begin{array}{l}14 \text { Atend. Ambulatoriais } \\
02 \text { Exam. Ambulatoriais } \\
14 \text { Exames por imagem } \\
07 \text { Exames laboratoriais }\end{array}$ \\
\hline $\begin{array}{l}23^{\circ} \text { BPM (Afog. da } \\
\text { Igazeira) }\end{array}$ & $\mathbf{0}$ & $\mathbf{0}$ & $\mathbf{0}$ & - & \\
\hline $\begin{array}{l}\text { CENTRO HOSPITALAR } \\
\text { J. MOURA SOARES }\end{array}$ & 0 & 0 & 0 & - & Não houve atend. este mês \\
\hline CLINICA SAME & 0 & 0 & 0 & - & Não houve atend. este mês \\
\hline $\begin{array}{c}\text { CENTRO DE ANÁLISES } \\
\text { CLÍNICAS MARIA DO } \\
\text { CARMO }\end{array}$ & 868,56 & 0 & 868,56 & - & 09 Exames laboratoriais \\
\hline $\begin{array}{l}\text { CLINICA SANTA } \\
\text { MARIA }\end{array}$ & $1.335,04$ & 428,65 & 906,39 & $\begin{array}{l}\text { Sem evolução médica }+ \text { sem fatura }+ \\
\text { sem biopsia aut. }\end{array}$ & $\begin{array}{c}09 \text { Atend. Ambulatoriais } \\
02 \text { internamento Hospitalar }\end{array}$ \\
\hline $\begin{array}{l}\text { LABORATÓRIO } \\
\text { MONICA MIRTES }\end{array}$ & 0 & 0 & 0 & - & Não houve atend. este mês \\
\hline $\begin{array}{c}\text { INST. DE OLHOS } \\
\text { Dr.JEOVÁ RAIMUNDO }\end{array}$ & 751,95 & 0 & 751,95 & - & 12 Atend. Ambulatoriais \\
\hline CLINICA SAMED & $1.083,32$ & 512,12 & 571,2 & Inter. Sem doc. Necessária a aud. & $\begin{array}{l}17 \text { Atend. Ambulatoriais } \\
01 \text { internamento Hospitalar }\end{array}$ \\
\hline \multicolumn{6}{|l|}{$4^{\circ}$ BPM (Petrolândia) } \\
\hline CLINICA SÃO GABRIEL & 0 & 0 & 0 & - & Não houve atend. este mês \\
\hline $\begin{array}{l}\text { HOSPITAL SÃO } \\
\text { THIAGO }\end{array}$ & $1.939,99$ & 90,42 & $1.849,57$ & $\begin{array}{l}\text { Ausência de laudo, valores diferentes } \\
\text { da tabela PMPE }\end{array}$ & $\begin{array}{l}15 \text { Atend Ambulatorial } \\
03 \text { Exames p/ Imagem } \\
05 \text { Exames ambulatoriais } \\
06 \text { Exames laboratoriais } \\
01 \text { Internamento }\end{array}$ \\
\hline $\begin{array}{l}\text { ALINE DE ALENCAR E } \\
\text { SÁ DA PENHA }\end{array}$ & 0 & 0 & 0 & - & Não houve atend. este mês \\
\hline $\begin{array}{l}\text { TOTAL GERAL EM } \\
\text { VALORES }(\mathrm{R} \$)\end{array}$ & $270.962,3$ & $11.019,52$ & $259.906,7$ & & \\
\hline $\begin{array}{l}\text { TOTALGERAL DE } \\
\text { CONTAS AUDITADAS } \\
\text { NO IINTERIOR }\end{array}$ & 0 & 0 & 0 & - & $\begin{array}{c}2461 \text { Contas atend. } \\
\text { Ambulatorial } \\
712 \text { Contas Ex. Imagem } \\
200 \text { Contas Ex. Diversos } \\
708 \text { Contas Ex. laboratoriais } \\
249 \text { Contas Internamento }\end{array}$ \\
\hline
\end{tabular}

Durante o período da pesquisa quantificou-se um total geral de contas pagas foi de R\$ 1.483.474,02 na capital e interior do estado de Pernambuco, deste foi gerada uma glosa de $\mathrm{R} \$$ 63.826,87, conforme pode ser visto na Tabela 3 . 
Id on Line Revista Multidisciplinar e de Psicologia

Id on Line Multidisciplinary and Psychology Journal

Tabela 3- Valor total de contas auditadas do Hospital da PMPE na capital e no interior no mês de agosto de 2010

\begin{tabular}{c|c|c|c|c|c}
\hline & Capital & Interior & Glosa & Valor total capital + interior & \\
\hline & & & & & 134 honorários médicos \\
TOTAL GERAL & & & & 4490 Contas atend. \\
PAGO CAPITAL + & $1.233,567,23$ & $259.906,79$ & $63.826,87$ & $\mathbf{R} \mathbf{1 . 4 8 3 , 4 7 4 , 0 2}$ & Ambulatorial \\
INTERIOR. (R\$) & & & & & 386 Contas Ex. Diversos \\
& & & & & 894 Contas Ex. laboratoriais \\
& & & & 366 Contas Internamento \\
\hline
\end{tabular}

Deste valor total é possível observar que neste período, na capital e no interior pernambucano, foram auditadas 4490 contas de atendimentos ambulatoriais, 2245 contas de exames por imagem, 366 contas de internamentos e 894 contas de exames laboratoriais, (Tabela 3). Neste tocante, é importante notar que, as contas do interior são obtidas somando todas as unidades de saúde do interior do estado onde existe convenio com o HPMPE, deste modo, as contas do interior só não ultrapassam as contas do Recife apenas no item exame por imagem onde o total de contas do interior é de 712 e da capital 1533 contas auditadas.

\section{Considerações Finais}

Observou-se no presente estudo que do total de cobranças, tivemos uma margem de 4,5\% de economia nos custos totais devido a eficiência do serviço de Auditoria. Para que possamos diminuir o percentual total de perdas hospitalares precisamos que enfermeiros e instituições avaliem como estão sendo realizados os registros de enfermagem, tendo uma visão crítica e propositora de sua atuação, pois devemos desempenhar as atividades focando sempre na qualidade da assistência prestada aos pacientes e o correto preenchimento da guia e do prontuário que são a principal demonstração disso.

Visto a quantidade de instituições conveniadas e o grande montante de contas reunidas para serem auditadas, é de extrema importância que estas contas estejam com todos os dados e contendo todas as informações pertinentes aos tratamentos realizados. Cabe à instituição de saúde atentar se realmente é vantajoso, economizar no quadro de profissionais, acarretando assim em glosas, ou se contratar mais profissionais seria a solução.

A questão de custos na área de saúde tem exigido profissionais cada vez mais qualificados que cooperem no resultado econômico da instituição. Dado que as perdas de materiais e medicamentos- as principais fontes lucrativas do hospital são pouco controladas, a auditoria em enfermagem pode desempenhar um trabalho proativo em semelhança a este aspecto 
Id on Line Revista Multidisciplinar e de Psicologia

Id on Line Multidisciplinary and Psychology Journal

Nesta pesquisa destaca-se a necessidade de que os enfermeiros passem a avaliar como estão fazendo os registros de sua equipe, fazendo um planejamento das atividades, visando educação continuada, realizando treinamentos sistematizados, capacitando sua equipe para a valorização das anotações.

\section{Referências}

BICHANGA, C.A.; BUD, M.H. Gerenciamento de custos através de avaliações de cobranças e sua efetividade. In: $7^{\circ}$ ENFTEC; 2000; São Paulo. Anais eletrônicos. São Paulo; 2000.

CAMELO, T.V.; SILVA JUNIOR, O.C. Auditoria de prontuários: um novo campo de atuação para enfermeiros. $13^{\circ}$ Seminário Nacional de Pesquisa em Enfermagem; 2005; São Luis (MA), Brasil.

Conselho Federal de Medicina. Código de ética dos profissionais de medicina [online]. Resolução 1331/89. Disponível em: www.portalmedico.org.br 2007.

D`INNOCENZO M.; FELDMAN, L.B.; FAZENDA, N.R.R.; HELITO, R.AB.; RUTHES, R.M. Indicadores, Auditorias, Certificações: Ferramentas de Qualidade para Gestão em Saúde. São Paulo (SP): Martinar, 2010.

FARACO, M.M.; ALBUQUERQUE, G.L. Auditoria do método de assistência de enfermagem. Rev. Bras. Enferm. 2004; 57(4): 421-4.

FERREIRA，T.S.; SOUZA-BRAGA，A.L.; CAVALCANTI-VALENTE， G.S..; SOUZA， D.F.; Carvalho-Alves, E.M. Auditoria De Enfermagem: O Impacto Das Anotações De Enfermagem No Contexto Das Glosas Hospitalares. Ano 9, v.9, n.1 Colômbia, 2009, 38-49, ISSN 1657-5997.

GOTO, D.Y.N. Instrumento de auditoria técnica de conta hospitalar mensurando perdas e avaliando a qualidade da assistência [monografia]. Curitiba: Universidade Federal do Paraná; 2001.

LOPES, C.M. Auditoria e distorções: ênfase nas atividades de anotações de enfermagem. Rev. Bras. Enferm. 1998; 51(1): 105-22.

MAIA, J.R.; PAES, P.P.L. Manual de Auditoria de contas Médicas. Hospital Geral de Juiz de Fora. 2005. Ministério da Defesa, Exército Brasileiro $4^{\mathrm{a}}$ Região Militar $/ 4^{\mathrm{a}}$ Divisão de Exército. Juiz de Fora, MG.

SCARPARO, A.F.; FERRAZ, C.A.; CHAVES, L.D.P.; ROTTA, C.S.G. Abordagem conceitual de métodos e finalidade da auditoria de enfermagem. Rev. Rene, Fortaleza, 2009. v. 10, n. 1, p. 1-165, jan./mar.

SILVA, S.H.; ORTIZ, D.C.F.; SHIMIZU, H.E.; TOTH, M. Auditoria em enfermagem: implantação e desenvolvimento no Hospital Universitário da Universidade de São Paulo. Rev. Esc. Enferm. USP 1990; 24(2): 1999-2009. 
Id on Line Revista Multidisciplinar e de Psicologia

Id on Line Multidisciplinary and Psychology Journal

Como citar este artigo (Formato ABNT):

CINTRA, G.M.G.; SOUZA, C.G.; SOUZA, M.G.S. Auditoria em Contas Médicas no Hospital da Polícia Militar de pernambuco. Id on Line Revista Multidisciplinar e de Psicologia, Julho de 2016, vol.10, n.30, Supl. 1, p. 92-106. ISSN 1981-1179.

Recebido: 10/04/2016

Aceito: 16/04/2016 